University of New Hampshire

University of New Hampshire Scholars' Repository

Physics Scholarship

Physics

4-2002

\title{
ULF waves in the solar wind as direct drivers of magnetospheric pulsations
}

L. Kepko

Harlan E. Spence

Boston University, harlan.spence@unh.edu

H. J. Singer

Follow this and additional works at: https://scholars.unh.edu/physics_facpub

Part of the Physics Commons

\section{Recommended Citation}

Kepko, L., H. E. Spence, and H. J. Singer, ULF waves in the solar wind as direct drivers of magnetospheric pulsations, Geophys. Res. Lett., 29(8), doi:10.1029/2001GL014405, 2002.

This Article is brought to you for free and open access by the Physics at University of New Hampshire Scholars' Repository. It has been accepted for inclusion in Physics Scholarship by an authorized administrator of University of New Hampshire Scholars' Repository. For more information, please contact Scholarly.Communication@unh.edu. 


\title{
ULF waves in the solar wind as direct drivers of magnetospheric pulsations
}

\author{
L. Kepko and H. E. Spence \\ Center for Space Physics, Boston University, Boston, Massachusetts, USA
}

\author{
H. J. Singer \\ NOAA Space Environment Center, Boulder, Colorado, USA
}

Received 19 November 2001; revised 5 February 2002; accepted 8 February 2002; published 19 April 2002.

[1] Global magnetospheric ULF pulsations with frequencies in the Pc 5 range $(f=1.7-6.7 \mathrm{mHz})$ and below have been observed for decades in space and on the Earth. Recent work has shown that in some cases these pulsations appear at discrete frequencies. Global cavity and waveguide modes have been offered as possible sources of such waves. In these models the magnetosphere is presumed to resonate globally at frequencies determined solely by its internal properties such as size, shape, field topology, mass density distribution, etc. We show in this work that upstream solar wind number density and dynamic pressure variations precede and drive compressional magnetic field variations at geosynchronous orbit. Furthermore, spectral analysis shows that wave power spectra in both the solar wind and magnetosphere contain peaks at the same discrete frequencies. Therefore, in contrast to the cavity mode hypothesis, we suggest that discrete ULF pulsations observed within the magnetosphere are at least sometimes directly driven by density oscillations present in the ambient solar wind. Finally, we comment on possible sources for such pulsations observed in the solar wind. INDEX TERMS: 2784 Magnetospheric Physics: Solar wind/magnetosphere interactions; 2752 Magnetospheric Physics: MHD waves and instabilities; 2740 Magnetospheric Physics: Magnetospheric configuration and dynamics

\section{Background}

[2] ULF pulsations with frequencies in the Pc5 range ( $f=1.7-$ $6.7 \mathrm{mHz}$ ) and below contribute to magnetospheric particle transport and diffusion and may play an important role in magnetospheric dynamics. For example, several studies have shown the existence of strong Pc5 wave power during the main phase of geomagnetic storms (e.g., Baker et al. [1998]; O'Brien et al. [2001]). This correlation has led some researchers to postulate that Pc5 and lower frequency pulsations provide the free energy needed to accelerate electrons to relativistic energies ( $\sim 0.5 \mathrm{MeV}$ and above) (e.g., Liu et al. [1999]; Elkington et al. [1999]). Others have associated longer period waves with substorm-related phenomena and tail dynamics [e.g. Sanchez et al., 1997].

[3] This paper concentrates on discrete ULF pulsations with frequencies $<3 \mathrm{mHz}$ observed at geosynchronous orbit and in the solar wind. There have been reports of ground-based and space-based observations of discrete ULF pulsations near $f=0.5$ $\mathrm{mHz}$ since the late 1960s. Herron [1967] produced a power spectrum of persistent geomagnetic variations which showed a peak centered near $0.5 \mathrm{mHz}$. More recently Chen and Kivelson [1991], using ISEE-2 magnetometer data from the magnetotail lobes, found a narrow-banded peak in power near $0.48 \mathrm{mHz}$.
Rinnert [1996] observed periodic enhancements with frequencies between 0.3 and $0.4 \mathrm{mHz}$ in EISCAT (European Incoherent SCATter) radar measurements of E-region electron density. Observations of gravity waves with similar frequencies were observed by Huang et al. [2000], during an extended period of northward IMF. They attributed the waves to a global cavity mode.

[4] Other papers have reported observations of pulsations with power at multiple discrete frequencies. For example, using Prognoz-8 magnetometer data from the magnetotail lobes, Nikutowski et al. [1996] found oscillations with $f=0.25,0.5,0.8$, and $1 \mathrm{mHz}$. A series of recent papers has focused on observations of spectral peaks near $f=1.3,1.9,2.6$, and $3.4 \mathrm{mHz}$ in high-latitude radar [Ruohoniemi et al., 1991; Samson et al., 1991; Walker et al., 1992; Fenrich et al., 1995] and ground magnetic field measurements [Samson et al., 1992a; Ziesolleck and McDiarmid, 1994; Francia and Villante, 1997]. The combination of the long period of these pulsations, the extended duration over which they were observed, and the nature of the discrete spectrum have led some researchers to suggest a global cavity or waveguide mode. In particular, Harrold and Samson [1992] and Samson et al. [1992b] have suggested that the discrete frequencies listed above were the result of a flankside waveguide mode. In this scenario compressional waves excited by a broadband impulse spectrum are confined between the magnetopause and an internal turning point, perhaps the plasmapause. Because these are bounded standing waves, the resonant frequencies generated are discrete. Waveguide modes are able to produce the low frequencies observed, and if the cavity is highly reflective these oscillations can last many cycles.

[5] Though it is well known that changes in solar wind dynamic pressure directly affects the magnetospheric field, few studies have explored the possibility that such discrete magnetospheric pulsations might arise directly from oscillatory sources inherent in the solar wind (see e.g., Matsuoka et al. [1995]; Korotova and Sibeck [1995]; Sarafopolous [1995]). Using data from the WIND spacecraft, we offer direct evidence that solar wind number density and ram pressure fluctuations observed far upstream from the terrestrial magnetosphere contain power at discrete frequencies. We show that these same oscillations are observed in the magnetosphere by the geosynchronous GOES spacecraft. The time-series, when appropriately time-shifted, are highly correlated and the power spectral peaks show excellent agreement. Based on these observations, we suggest that the solar wind is in some cases a direct source for discrete ULF pulsations.

\section{Events}

\subsection{February 5, 2000}

[6] The Wind spacecraft was located in the upstream solar wind at $(90,-28,-1) R_{E}$ in GSM coordinates. A five-hour interval of number density and dynamic pressure measurements $\left(n V^{2}\right)$ from 


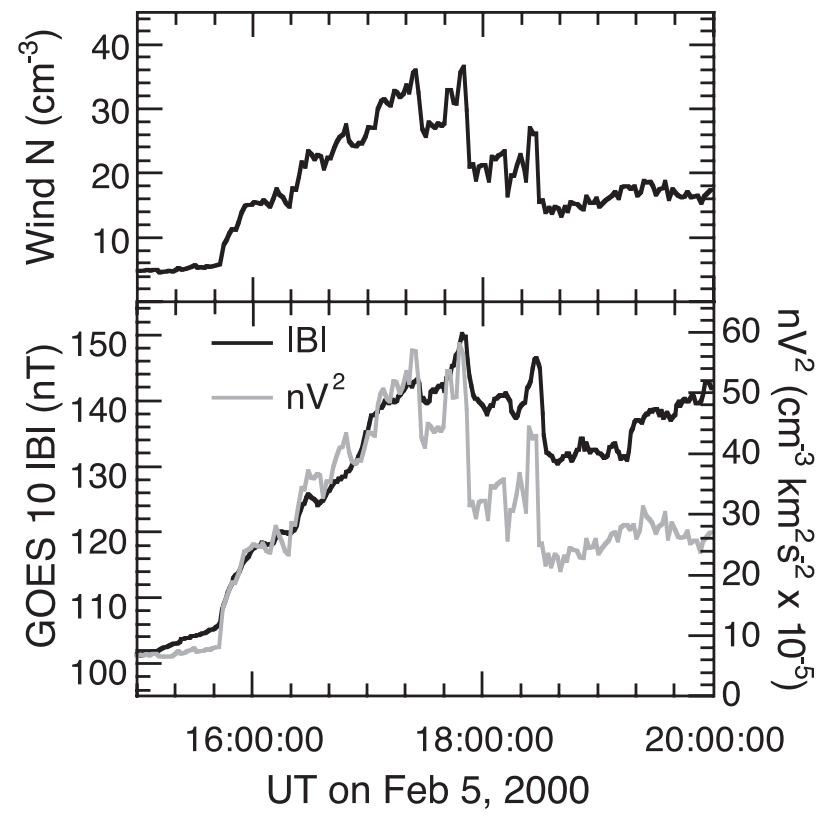

Figure 1. (a) Solar wind number density and (b) solar wind dynamic pressure, $n V^{2}$ (grey), and geosynchronous magnetic field (black) measurements from February 5, 2000. The solar wind data have been time-shifted by $+17 \mathrm{~min}$.

the Solar Wind Experiment (SWE) [Ogilvie et al., 1995] are shown in Figure 1. We note that the time-series of dynamic pressure is qualitatively similar to the number density; that is, changes in number density are principally responsible for changes in dynamic pressure. The magnetic and thermal pressures calculated from the Wind measurements (not shown) are highly anti-correlated $(R=$ $-0.9)$. We also show the magnitude of the magnetic field measured by the geosynchronous GOES-10 satellite. The total field is dominated by contributions from the $B_{z}$ component, consistent with the location of GOES near the geomagnetic equator. The oscillations are largely observed in $B_{z}$, indicating that the waves were mostly compressional. The Wind data have been shifted by +17 min to align the dynamic pressure structures in the solar wind with the magnetic variations at GOES. The computed correlation coefficient $R=0.9$ of the Wind dynamic pressure and GOES 10 magnetic field measure-

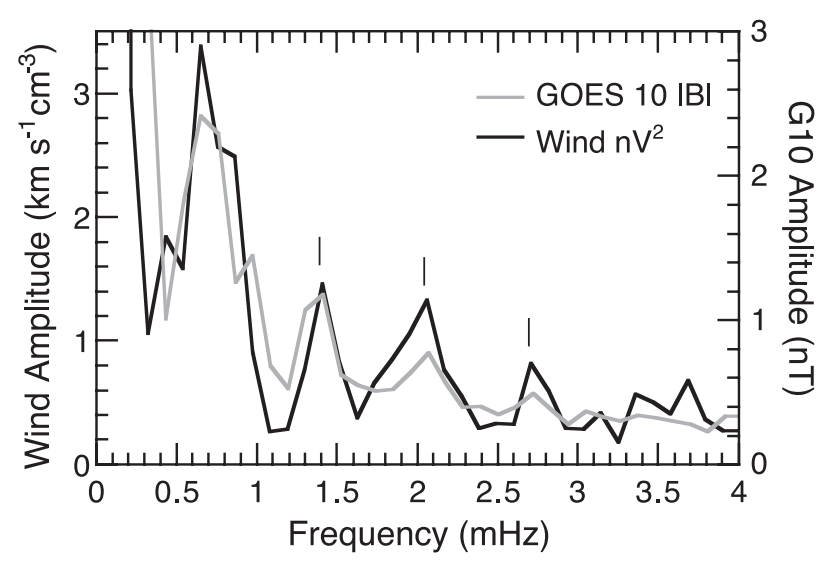

Figure 2. Fourier transforms of the solar wind dynamic pressure $\left(n V^{2}\right)$ and geosynchronous magnetic field strength from GOES 10 for the period 1545-1830 UT on February 5, 2000.

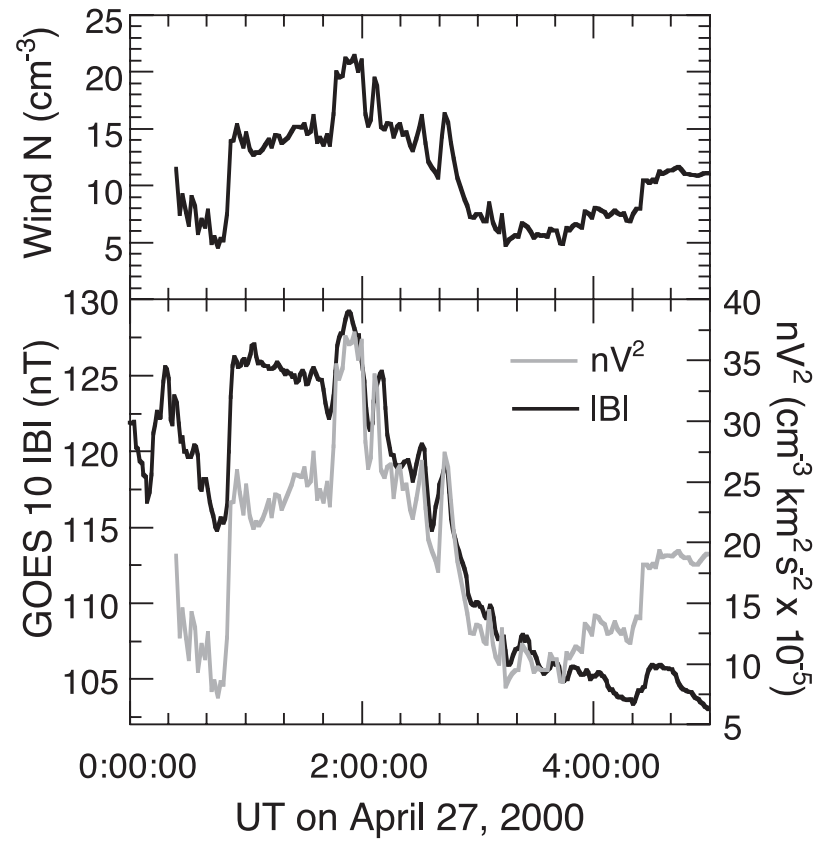

Figure 3. (a) Solar wind number density and (b) solar wind dynamic pressure, $n V^{2}$ (grey), and GOES 10 magnetic field strength (black) measurements for April 27, 2000.

ments is high and significant. The time delay from Wind to the magnetosphere calculated using the upstream position and the measured solar wind velocity $\left(V_{x} \sim 400 \mathrm{~km} / \mathrm{s}\right)$ is $\sim 22 \mathrm{~min}$, which is consistent with the time determined by the crosscorrelation analysis.

[7] While it is clear that the solar wind dynamic pressure and magnetospheric field variations are similar, it is not quite so obvious that the observed variations occur at discrete frequencies. We have calculated the Fourier transforms of both signals from 1545-1830 UT, with the Wind data interval 17 minutes earlier to account for propagation to the magnetosphere. The spectral estimates, with $\Delta f=0.11 \mathrm{mHz}$, are shown in Figure 2. Significant power at frequencies of $f=0.7,1.4,2.0$, and $2.7 \mathrm{mHz}$ are evident in both the solar wind dynamic pressure and GOES $10|B|$. Neither altering the width of the window $(\sim \pm 10 \%)$ over which the transform is computed nor moving the window in time ( $\pm 10 \mathrm{~min})$ alter the spectra significantly. We therefore believe these to be robust peaks.

\subsection{April 27, 2000}

[8] A second example occurred while Wind was located in the solar wind at $(84,17,0) R_{E}$ in GSM coordinates. As in the previous example, we plot in Figure 3 the time-shifted solar wind number density and dynamic pressure measured by SWE onboard Wind and the total strength of the magnetic field measured by GOES 10 . The Wind data are plotted with a time-shift of $+22 \mathrm{~min}$, which gives good visual agreement between variations in the solar wind dynamic pressure and geosynchronous magnetic field. Ballistic propagation yields a similar time-shift. The magnetic and thermal pressures calculated from the Wind measurements are again highly anti-correlated $(R=-0.94)$.

[9] As in the previous example, variations in the solar wind dynamic pressure (again driven largely by number density variations) and the geosynchronous magnetic field are highly correlated $(R=0.9)$. We chose the interval $0152-0500$ UT (Wind data offset by -22 min from that window), which avoids the discontinuous jumps in dynamic pressure and magnetic field strength, over which to obtain our spectral estimates. The results 


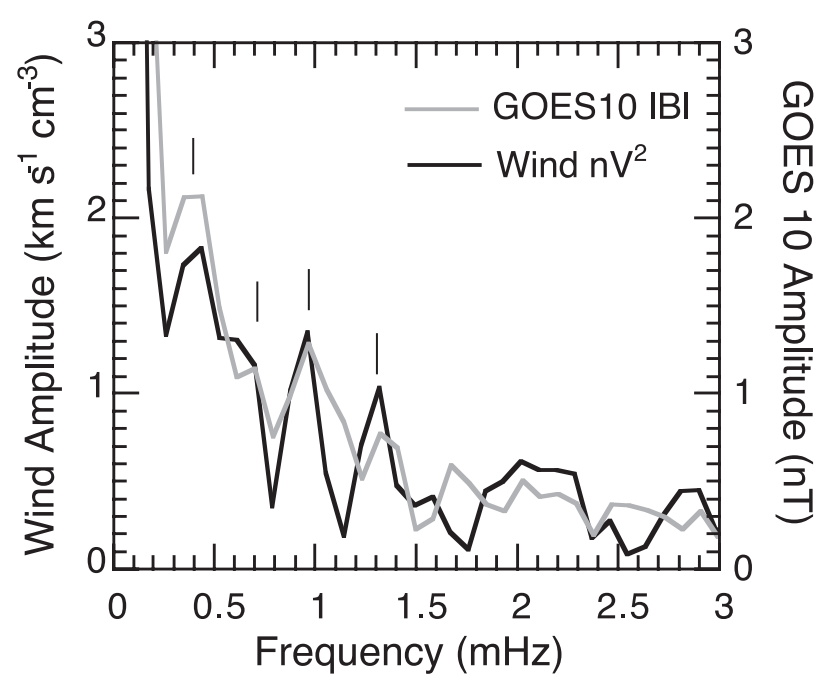

Figure 4. Fourier transforms of WIND solar wind dynamic pressure $\left(n V^{2}\right)$ and GOES 10 magnetic field strength measurements for the period 0152-0500 UT on April 27, 2000.

of the Fourier transform are shown in Figure 4. Significant spectral peaks at $f=0.4,0.7,1.0$, and $1.3 \mathrm{mHz}$ are observed in both data sets.

\section{Discussion and Conclusions}

[10] We have presented two events in which both the correlation of the time-series and the spectral properties of the solar wind dynamic pressure match those of geosynchronous magnetic field measurements. Power spectra of both data sets show enhanced power at discrete spectral peaks at frequencies $<2-3 \mathrm{mHz}$. Some of the higher-frequency peaks match those reported by Samson et al. $[1992 \mathrm{a}, 1992 \mathrm{~b}]$ and other investigations and attributed to a magnetospheric waveguide, as reviewed in the introduction. The quality of the correlations in the time domain $(R \sim 0.9)$ as well as the robustness of the spectral peaks give us confidence that the power is physically meaningful, and not an artifact of the analysis. As reviewed in our introduction, there have been previous reports of observations of magnetospheric oscillations with frequencies similar to what we have observed here. However, this is the first time that such low frequency oscillations have been shown to have a source in the upstream solar wind.

[11] The high-correlation between solar wind dynamic pressure variations far upstream and magnetospheric oscillations precludes a cavity or waveguide explanation. Instead, we suggest that the ULF oscillatory changes in solar wind dynamic pressure slowly affect the size of the magnetospheric cavity. The magnetospheric field increases or decreases as needed to balance the internal magnetic pressure against the external dynamic pressure. In this manner, oscillatory variations of the dynamic pressure lead directly to oscillatory changes in the magnetospheric magnetic field strength. As long as these changes are slow with respect to both the Alfvénic travel time through the system $(\sim 3 \mathrm{~min})$ and the time needed for the dynamic pressure disturbance to convect past the Earth ( $\sim 5 \mathrm{~min})$, than the problem is quasi-static. The shortest period observed in this paper is $\sim 6 \mathrm{~min}$, with the majority of the power at periods of 10-30 min. It is, in a sense, a forced breathing of the magnetosphere.

[12] A solar wind source for magnetospheric pulsations at frequencies $<2-3 \mathrm{mHz}$, while potentially solving a number of outstanding questions, also raises new questions. For example, are the pulsations observed in this work related to the $f=1.3,1.9,2.6$, 3.4, and $4.2 \mathrm{mHz}$ frequencies reported by Samson et al. [1991] and others? These frequencies have been attributed to a global wave- guide mode [Samson et al., 1992b]. Although the results here do not disprove the waveguide model, the overlap of our observed frequencies near 1.3, 1.9 and $2.7 \mathrm{mHz}$ suggest a reexamination of the source of the higher frequencies. Also, are the waves observed here related to the ULF waves that have been postulated to energize electrons to $\mathrm{MeV}$ energies during geomagnetic storms? For this to occur, the waves would have to be present for many hours in the post-shock solar wind or during high-speed streams. Whether this is true or not is an area that will be explored in future work.

[13] The events presented in this work were selected based on visual inspection of the number density variations in the solar wind which facilitated correlation with geosynchronous measurements. We do not at this time have an estimate on how often such ULF waves are observed in the solar wind or what fraction of magnetospheric pulsations are directly driven by these waves. We are currently working to quantify these numbers, and will present the results in a future paper.

[14] We note that although we have identified a relationship between solar wind and magnetospheric ULF pulsations, what ultimately determines the frequency of the solar wind dynamic pressure variations remains unanswered. Sibeck and Newell [1989] argued that $\sim 2 \mathrm{mHz}$ dynamic pressure fluctuations in the magnetosheath observed simultaneously with magnetic perturbations within the magnetosphere were generated at the bow shock. In this study, however, the solar wind observations are far enough upstream that we believe it precludes a source centered on the bow shock. Instead, the variations must be a feature inherent in the solar wind.

[15] One possibility for the source of the spectrum of solar wind number density and dynamic pressure oscillations is that it results from an instability generated in the solar wind during its passage through interplanetary space. We can at this time offer no suggestion as to what this instability might be, however, and it is unclear how an instability could generate such stable, reproducible frequencies. A second possibility is that the frequencies are the result of solar oscillations. There has been considerable interest in this topic after it was suggested by Thomson et al. [1995] that discrete power in solar wind energetic particles and magnetic fields measured by the Ulysses spacecraft were similar to known gravity (g) and pressure (p) mode frequencies of the sun. The p modes of the sun are in the range of $1-5 \mathrm{mHz}$, the same interval as the waves reported in this paper. Though relating terrestrial ULF pulsations to solar oscillations is an intriguing possibility, subsequent work addressing whether the solar wind contains imprints of solar modes has yielded conflicting results [Roberts et al., 1996; Thomson et al., 1996; Paulerena, 1996; Riley and Sonett, 1996; Thomson et al., 2001], and the issue remains unresolved.

[16] Acknowledgments. We thank K. W. Ogilvie, A. J. Lazarus, and J. C. Kasper for providing the WIND SWE data. We also thank M. Lessard, M. Hudson, H. Petschek and N. Crooker for helpful discussions. Work at Boston University was supported by NASA grant NAG5-7706.

\section{References}

Baker, D. N., T. I. Pulkkinen, X. Li, S. G. Kanekal, J. B. Blake, R. S. Selesnick, M. G. Henderson, G. D. Reeves, H. E. Spence, and G. Rostoker, Coronal mass ejections, magnetic clouds, and relativistic magnetospheric electron events: ISTP, J. Geophys. Res., 103, 17,279, 1998.

Chen, S., and M. G. Kivelson, On ultralow frequency waves in the lobes of the Earths magnetotail, J. Geophys. Res., 96, 15,711, 1991.

Elkington, S. R., M. K. Hudson, and A. Chan, Acceleration of relativistic electrons via drift-resonant interaction with toroidal-mode Pc-5 ULF oscillations, Geophys. Res. Lett., 26, 3273, 1999.

Fenrich, F. R., J. C. Samson, G. Sofko, and R. A. Greenwald, ULF highand low-m field line resonances observed with the Super Dual Auroral Radar Network, J. Geophys. Res., 100, 21,535, 1995.

Francia, P., and U. Villante, Some evidence of ground power enhancements at frequencies of global magnetospheric modes at low-latitudes, Ann. Geophysicae, 15, 12, 1997. 
Harrold, B. G., and J. C. Samson, Standing ULF modes of the magnetosphere: A theory, Geophys. Res. Lett., 19, 1811, 1992.

Herron, T. J., An average geomagnetic power spectrum for the period range 4.5 to 12,900 seconds, J. Geophys. Res., 72, 759, 1967.

Huang, C.-S., G. J. Sofko, A. V. Kustov, J. W. MacDougall, D. A. Andre, W. J. Hughes, and V. O. Papitashvili, Quasi-periodic ionospheric disturbances with a 40-min period during prolonged northward interplanetary magnetic field, Geophys. Res. Lett., 27, 1795, 2000.

Korotova, G. I., and D. G. Sibeck, A case study of transient event motion in the magnetosphere and in the ionosphere, J. Geophys. Res., 100, 35, 1995.

Liu, W. W., G. Rostoker, and D. N. Baker, Internal acceleration of relativistic electrons by large-amplitude ULF pulsations, J. Geophys. Res., 104, 17,391, 1999

Matsuoka, H., K. Takahashi, K. Yumoto, B. J. Anderson, and D. G. Sibeck, Observation and modeling of compressional Pi 3 magnetic pulsations, J. Geophys. Res., 100, 12,103, 1995.

Nikutowski, B., J. Bchner, S. Klimov, S. Romanov, A. Petrukovich, and S. Savin, Long periods of the ulf wave activity in the Earths magnetotail lobes, Adv. Space. Res., 18, 55, 1996.

O’Brien, T. P., R. L. McPherron, D. Sornette, G. D. Reeves, R. Friedel, and H. J. Singer, Which magnetic storms produce relativistic electrons at geosynchronous orbit?, J. Geophys. Res., 106, 15,533, 2001.

Ogilvie, K. W., D. J. Chorney, R. J. Fitzenreiter, F. Hunsaker, J. Keller, J. Lobell, G. Miller, J. D. Scudder, E. C. Sittler Jr., R. B. Torbert, D Bodet, G. Needell, A. J. Lazarus, J. T. Steinberg, J. H. Tappan, A. Mavretic, and E. Gergin, SWE, a comprehensive plasma instrument for the Wind spacecraft, Space Sci. Rev., 71, 55, 1995.

Riley, P., and C. P. Sonett, Interplanetary observations of solar g-mode oscillations?, Geophys. Res. Lett., 23, 1541, 1996.

Rinnert, K., Quasi-periodic precipitation with periods between 40 and 60 minutes, Ann. Geophys., 14, 707, 1996.

Roberts, D. A., K. W. Ogilvie, and M. L. Goldstein, Scientific correspondence, Nature, 381, 31, 1996.

Ruohoniemi, J. M., R. A. Greenwald, K. B. Baker, and J. C. Samson, $\mathrm{HF}$ radar observations of Pc 5 field line resonances in the midnight early morning MLT sector, J. Geophys. Res., 96, 15,697, 1991.
Sanchez, E. R., J. D. Kelly, V. Angelopoulos, T. Hughes, and H. Singer, Alfvn modulation of the substorm magnetotail transport, Geophys. Res. Lett., 24, 979, 1997.

Sarafopolous, D. V., Long duration Pc 5 compressional pulsations inside the Earths magnetotail lobes, Ann. Geophys., 13, 926, 1995.

Thomson, D. J., C. G. Maclennan, and L. J. Lanzerotti, Propagation of solar oscillations through the interplanetary medium, Nature, 376, 139, 1995.

Thomson, D. J., C. G. Maclennan, and L. J. Lanzerotti, Scientific correspondence, Nature, 371, 32, 1996.

Thomson, D. J., L. J. Lanzeroti, and C. G. Maclennan, Interplanetary magnetic field: Statistical properties and discrete modes, J. Geophys. Res., 106, 15,941, 2001.

Samson, J. C., R. A. Greenwald, J. M. Ruohoniemi, T. J. Hughes, and D. D. Wallis, Magnetometer and radar observations of magnetospheric cavity modes in the Earths magnetosphere, Can. J. Phys., 69, 929, 1991.

Samson, J. C., D. D. Wallis, T. J. Hughes, F. Creutzberg, J. M. Ruohoniemi, and R. A. Greenwald, Substorm intensifications and field line resonances in the nightside magnetosphere, J. Geophys. Res., 97, 8495, 1992a.

Samson, J. C., B. G. Harrold, J. M. Ruohoniemi, R. A. Greenwald, and A. D. M. Walker, Field line resonances associated with MHD waveguides in the magnetosphere, Geophys. Res. Lett., 19, 441, 1992 b.

Walker, A. D. M., J. M. Ruohoniemi, K. B. Baker, R. A. Greenwald, and J. C. Samson, Spatial and temporal behavior of ULF pulsations observed by the Goose Bay HF radar, J. Geophys. Res., 97, $12,187,1992$.

Ziesolleck, C. W. S., and D. R. McDiarmid, Auroral latitude Pc 5 field line resonances: Quantized frequencies, spatial characteristics, and diurnal variations, J. Geophys. Res., 99, 5817, 1994.

L. Kepko and H. E. Spence, Center for Space Physics, Boston University, 725 Commonwealth Ave., Boston, MA 2215 USA. (1kepko@bu.edu; spence@bu.edu)

H. J. Singer, Space Environment Center, NOAA, Boulder, CO 80303 , USA. (Howard.Singer@noaa.gov) 